\title{
Anterior cruciate ligament tears treated with percutaneous injection of autologous bone marrow nucleated cells: a case series
}

This article was published in the following Dove Press journal:

Journal of Pain Research

31 July 2015

Number of times this article has been viewed

\author{
Christopher J Centeno ${ }^{1,2}$ \\ John Pitts' \\ Hasan Al-Sayegh ${ }^{2}$ \\ Michael D Freeman ${ }^{3}$ \\ 'Centeno-Schultz Clinic, ${ }^{2}$ Regenerative \\ Sciences, LLC., Broomfield, CO, \\ ${ }^{3}$ Public Health Department, Oregon \\ Health and Science University, \\ Portland, OR, USA
}

Introduction: This was a prospective case series designed to investigate treatment for anterior cruciate ligament (ACL) tears using an injection of autologous bone marrow concentrate.

Methods: Consecutive adult patients presenting to a private outpatient interventional musculoskeletal and pain practice with knee pain, ACL laxity on exam, and magnetic resonance imaging (MRI) evidence of a grade 1, 2, or 3 ACL tears with less than $1 \mathrm{~cm}$ retraction were eligible for this study. Eligible patients were treated with an intraligamentous injection of autologous bone marrow concentrate, using fluoroscopic guidance. Pre- and postprocedural sagittal MRI images of the ACLs were analyzed using ImageJ software to objectively quantify changes between pre- and posttreatment scans. Five different types of measurement of ACL pixel intensity were examined as a proxy for ligament integrity. In addition pain visual analog scale (VAS) and Lower Extremity Functional Scale (LEFS) values were recorded at baseline and at 1 month, 3 months, 6 months, and annually postinjection. Objective outcomes measured were pre- to post-MRI measurement changes, as analyzed by the ImageJ software. Subjective outcomes measured were changes in the VAS and LEFS, and a self-rated percentage improvement.

Results: Seven of ten patients showed improvement in at least four of five objective measures of ACL integrity in their postprocedure MRIs. In the entire study group, the mean gray value, median, raw integrated density, and modal gray value all decreased toward low-signal ACLs ( $P=0.01, P=0.02, P=0.002$, and $P=0.08$ ), indications of improved ligament integrity. Seven of ten patients responded to the self-rated metrics follow up. The mean VAS change was a decrease of $1.7(P=0.25)$, the mean LEFS change was an increase of $23.3(P=0.03)$, and mean reported improvement was $86.7 \%$.

Conclusion: Based on this small case series, autologous bone marrow concentrate shows promise in the treatment of grade 1,2, and possibly grade 3 ACL tears without retraction. Further investigation using a controlled study design is warranted.

Keywords: anterior cruciate ligament injury, regenerative therapy, bone marrow concentrate, mesenchymal stem cells, knee instability

\section{Introduction}

The anterior cruciate ligament (ACL) is a commonly injured knee ligament in the United States population, and accordingly, there are more than 150,000 ACL surgical reconstruction procedures performed per year. ${ }^{1}$ The ACL can be torn completely, which has a clear natural history of resulting in long-term instability in $15 \%-66 \%$ of patients, or be partial torn, in which the natural history is less clear. ${ }^{2}$ A review by Pujol et al showed that partial ACL tears could have a favorable short- to medium-term outcome with nonoperative management if sports activity was limited. Surgical indications for
Correspondence: John Pitts Centeno Schultz Clinic, 40I Summit Blvd, Suite 20I, Broomfield, CO 80022, USA

$\mathrm{Tel}+\mathrm{I} 3034296448$

Fax +l 3034296373

Email jpitts@centenoschultz.com 
both partial and complete ACL ruptures are continued instability, or ligament injury and returning to pivoting sport. ${ }^{3}$

Although ACL reconstruction can have good success rates, one of the potential adverse outcomes following ACL reconstruction is lack of restoration of full physiologic movement, due to the biomechanically disadvantageous location of the graft placement. ${ }^{4}$ This leads to increased tibial rotation and impaired neuromuscular control during high-demand activities among patients with ACL grafts, ${ }^{4}$ potentially resulting in increased cartilage load and thus higher risk of reinjury. For these and other reasons, although surgical reconstruction remains a standard and common procedure for management of ACL tears, alternative treatments are needed that can both address the integrity of the ligament and at the same time maintain the biomechanical competence of the knee joint.

Regenerative therapy of some select ACL injuries is one such possible treatment. Regenerative therapy, a cell-based technique directed at improved healing of the injured ligament, carries with it the potential of preserving the proprioceptive nerve fibers and collagenous architecture of the ligament, which in turn, preserves the normal biomechanics of the knee joint. ${ }^{5}$ Several experimental techniques aimed at regeneration of the ACL have been described in the literature. The application of extracellular matrix derived from pigs, combined with suture repair of torn ACLs, has been shown to accelerate healing of the ligament versus suture repair alone in a goat model. ${ }^{6}$ In other animal studies, partial ACL tears treated with platelet rich plasma (PRP) combined with a collagen matrix demonstrated healing and improved function in comparison with a control group. ${ }^{7}$ Seijas et al reported high return to sport in professional football players with a partial ACL tear treated with surgical reconstruction of the torn bundle and intraligamentous placement of platelet-derived growth factors into the intact bundle. ${ }^{2}$ Other in vitro and in vivo animal models have demonstrated neovascularization, granulation, and stimulation of mesenchymal stem cells (MSCs), such as fibroblasts, following the application of various growth factors and PRP to torn ACLs. ${ }^{7}$ Additional animal studies have described intraarticular (IA) injection of bone marrow-derived MSCs, with promising results for repair of ACL tears. ${ }^{7,8}$

There may be merit in healing ACL tears in situ. First, ACL grafts are placed in a nonphysiologic position, which could impact normal biomechanics. ${ }^{9}$ Second, osteoarthritis is common after ACL reconstruction. ${ }^{10}$ One possible benefit from MSCs is that they may aid in cartilage repair. ${ }^{11}$ Finally, proprioception is often lost after ACL repair, ${ }^{12}$ which may be due to the fact that the ligament graft doesn't contain viable or intact proprioceptors. An in site repair may allow for retention of intact proprioceptors, leading to better neuromuscular control. These principles may translate to an effective clinical application, but as of the date of the present study, there is a paucity of evidence for MSC therapy as a treatment for ACL injuries in humans. Adult MSCs have been demonstrated as safe, multipotent therapeutic agents in regenerative medicine, including for a number of orthopedic applications. ${ }^{13}$ We have previously published longitudinal studies demonstrating the safety and efficacy of MSCs to treat knee osteoarthritis. ${ }^{14,15}$

In the present study, we describe a treatment for magnetic resonance imaging (MRI)-documented ACL tears in a case series of adults using bone marrow concentrate (BMC) rich in MSCs, in which therapeutic efficacy was assessed by pain and functional outcome measures as well as MRI evidence of structural alteration of the ACL. This is an introductory study on the use of regenerative therapies for treatment of different ACL injuries. We believe there is a subset of patients who may benefit from regenerative therapies, such as patients with nonhealing grade 1-2 tears for whom surgery may not be recommended or patients with grade 3 tears without retraction of the ligament fibers who are surgical candidates but wish to avoid surgery. Regenerative therapies in the former subset of patients would likely be more effective. Based on the work of Rudolph et al, patients with more normal knee kinematics after ACL rupture (copers) may perform better than those with functional instability (noncopers). ${ }^{16}$ With this study, we hoped to gain insight on the safety and preliminary efficacy for this treatment, as well as provide the foundation for future more rigorous investigation.

\section{Methods and materials}

Consecutive patients presenting to an interventional pain practice with complaints of knee instability with or without pain and with an ACL tear previously documented with MRI, and laxity with Lachman testing ${ }^{17}$ on exam were enrolled in a case series of the first set of ACL treatments, under a larger registry of all MSC treatments tracked by our clinic. Patients were not paid for their participation. Patients were included in the analysis of prospectively collected registry data if they had a grade 1, 2, or 3 ACL tear without greater than $1 \mathrm{~cm}$ retraction, and agreed to participate. We defined retraction as any visible area of increased MRI signal intensity within the substance of the ligament that was full thickness and resulted in a discontinuity of the course of the structure. To our knowledge, this is the first study of ACL tears to focus on the extent of the separation of torn ACL fragments, thus this delineation has not been validated previously. Patients treated during acute ( $<1$ month postinjury), subacute injury 
(1-6 months postinjury), or in a chronic state of injury ( $>6$ months postinjury $)^{18}$ were eligible for inclusion. Exclusion criteria were grade 3 ACL tear with $>1 \mathrm{~cm}$ retraction, active neoplasm within the past 5 years, a history or presence of anemia, or age younger than 16 years. Patients provided consent verbally and in writing. Clinical outcomes for pain and function were recorded at baseline and prospectively at 1 month, 3 months, 6 months, and annually following treatment. Additionally, percentage improvement on a Likert scale was recorded at 1 month, 3 months, 6 months, and annually following treatment. Institutional review board oversight and approval was provided by the International Cellular Medicine Society.

ACL injuries were graded as follows: ${ }^{19}$

- Grade 1 sprain: the ligament is partial torn, with less than half of the ligament substance disrupted

- Grade 2 sprain: the ligament is partially torn, with more than half of the ligament substance disrupted

- Grade 3 sprain: the ligament is completely torn.

\section{Treatment protocol}

\section{Preinjection}

The first step of the treatment was a preinjection of a hypertonic dextrose solution into the ACL 2-5 days prior to injection of the BMC. The purpose of this preinjection procedure was to introduce a chemical irritant to the ACL in order to prompt a brief inflammatory response. A 25 gauge 3.0 inch Quinke needle (catalog number 405170; BD Biosciences, Franklin Lakes, NJ, USA) was inserted through the skin overlying the patellar tendon and directed through the inferior patellar tendon to a location just anterior to the tibial spine, on lateral fluoroscopy. Midline needle placement was confirmed on the anterior posterior fluoroscopic view. Iodixanol (Visipaque $^{\mathrm{TM}}$ NDC 0407-2223-06; GE Healthcare, Little Chalfont, UK) radiographic contrast was injected to confirm flow in the ACL sheath traveling between the radiographic origin and insertion landmarks, in both views (Figure 1). This was followed by injection of $3-5 \mathrm{~mL}$ of $12.5 \%$ dextrose (NDC 0409-6648-02) and 0.1\% lidocaine (NDC 0409-4276-02) in normal saline (NDC 0409-4888-50).

\section{Harvest and concentration of bone marrow aspirate}

The next step of the treatment was to harvest bone marrow and isolate the portion containing MSCs from each patient, in preparation for reinjection. Prior to the procedure, the patients were restricted from taking corticosteroids and nonsteroidal anti-inflammatory drugs (NSAIDs) for at least 2 weeks, as these medications can impair soft tissue healing. ${ }^{20-24}$

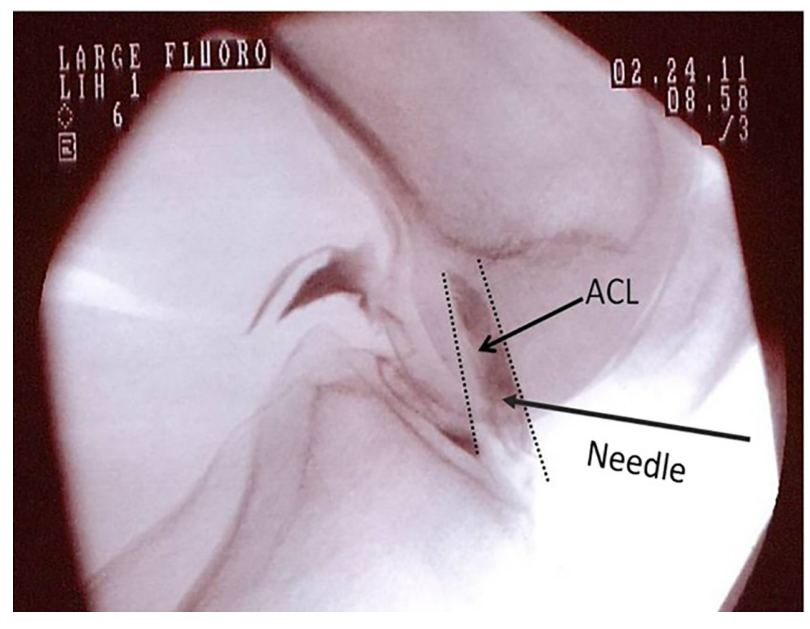

Figure I ACL injection with fluoroscopy. Abbreviation: $\mathrm{ACL}$, anterior cruciate ligament.

Whole bone marrow isolate was harvested from the patients' iliac crest, under ultrasound or fluoroscopic guidance. Approximately $10-15 \mathrm{~mL}$ of bone marrow aspirate was drawn from six sites into heparinized syringes. There were 1,000 units of heparin (NDC 25021-403-01 and 25021-404-01) per $1 \mathrm{~mL}$ of volume collected in the syringe. The aspirate was processed by hand, in a sterile ISO-7-class clean room and in ISO-5-class laminar flow cabinets, to isolate the buffy coat through centrifugation. This isolation produced $1-5 \mathrm{~mL}$ of BMC, which was then taken via sterile transport over the short distance back to the operating room. The nucleated cell count of the injectate was counted and recorded by lab staff with a cell counter (TC10; Bio-Rad Laboratories, Hercules, CA, USA) via light microscopy. Coincident with this harvest procedure, approximately $60 \mathrm{~mL}$ of heparinized venous blood was drawn to be used for isolating PRP and platelet lysate (PL). To prepare the PRP, plasma was prepped via centrifugation at $200 \times g$ to separate the plasma and buffy coat layers from the red blood cells. The resultant liquid lying above the concentrated solids (supernatant) was red cell/white cell-poor. To prepare the PL, PRP was drawn off and stored at $-20^{\circ} \mathrm{C}$ overnight; platelets were recentrifuged, and the supernatant drawn off. The purpose of freezing was to initiate lysis of the platelets. No commercial automated systems were used. All samples were processed in a current good manufacturing practice (cGMP) air-handling lab, using "off the shelf" equipment and a dedicated lab staff.

\section{Reinjection of the BMC}

Needle placement into the ACL was accomplished utilizing the same procedural protocol described for the preinjection. The injectate consisted of 2-3 mL of BMC, PRP, and PL, 
and was injected directly into the ligament. The needle was withdrawn from the ligament approximately $1 \mathrm{~cm}$, and while still in the joint, approximately $2-4 \mathrm{~mL}$ of a mixture of $1 \mathrm{~mL}$ of PRP, $1 \mathrm{~mL}$ of PL, and any remaining BMC were injected into the joint.

After the procedure, the patients were given instructions to participate in activity as tolerated. No posttreatment bracing was administered. All of the patients were encouraged to participate in physical therapy, but this was not required nor controlled. A physical therapy prescription was given if asked.

\section{Outcome measures}

Patients were enrolled in a treatment registry and tracked prospectively via an electronic database system using ClinCapture software (Clinovo Clinical Data Solutions, Sunnyvale, CA, USA) (http://www.clinovo.com/clincapture). The program includes an automated emailing system to send patients clinical outcome questionnaires to complete. A pain visual analog scale (VAS) and the Lower Extremity Functional Scale (LEFS) ${ }^{25}$ were recorded preoperatively (same day as the procedure) and postoperatively. Postoperatively, matching questionnaires were sent at 1 month, 3 months, 6 months, 12 months, and 24 months. In addition, a subjective percentage-improvement Likert-scale (from -90\% worsened to $100 \%$ improved) was recorded postoperatively. The last recorded follow ups were reported. During the study period, the functional questionnaires for gathering patient follow-up data were updated as part of changes to the treatment registry, and thus for the first two patients, the pretreatment functional data were recorded using the Functional Rating Index (FRI), ${ }^{26}$ whereas posttreatment function was recorded using the LEFS. However, the Likert improvement and the complications questions were the same throughout the study period.

\section{Imaging analysis}

Patients received a MRI scan of the knee, prior to treatment and at a minimum of 3 months posttreatment. The model and field strength of the MRI scanners as well as the available sequences varied between patients; however, for each patient, the pre- and posttreatment scans were always performed on the same scanner and with the same sequences. To quantify and reduce the variability in the interpretation of the changes in the MRI appearance of the ACL ligaments, we used computerized pixel analysis, using Image J software. Image J is a public Java image processing and analysis program developed at the National Institutes of Health (NIH) (http:// rsbweb.nih.gov/ij/). We chose metrics that the software could produce that objectively measure gray scale. The metrics used to assess the appearance of the ACL were the mean gray value, modal gray value, median, skewness, and raw integrated density.

Once both pre- and posttreatment images were obtained, the image selected for the pretreatment ligament integrity assessment was the sagittal T1 weighted, proton density (PD)weighted ACL sequence, PD sequence, PD fast spin-echo sequence, or the PD fat saturation sequence - whichever image visualized the greatest cross-sectional area of the ACL. For the posttreatment analysis, the closest matching image was selected from the same imaging sequence type. The same MRI scan sequence was selected for each individual for both studies. If a single sagittal image was insufficient to capture a complete slice through the ACL, two adjoining images were used for analysis. All the selected images were saved in a JPEG format.

\section{Imaging assessment protocol}

A selected ACL image was opened with the ImageJ software. Utilizing the software, the examiner manually outlined the ACL to create a region of interest (ROI). The examiner was instructed to only outline where he believed the ACL to be on the image and that the posterior extent of the ROI should not be more posterior than the posterior margin of the femoral condyle. Image J created a histogram of pixel frequency and intensity as a proxy for ligament integrity (Figure 2). It also calculated the selected metrics: mean gray value, modal gray value, median, skewness, and raw integrated density.

First, an inter- and intrarater reliability study was performed. Three random ACL images were selected and then measured by three examiners (CJC, BN, and JP), with each examiner manually outlining the ACL using the protocol stated above. The examiners were blinded both as to whether the image was pre- or posttreatment, as well as regarding the results of the other examiners. The resulting three images were measured serially three times each, and then the results were assessed for intra- and interexaminer agreement. The averages of the three serial measurements for each image were used for the statistical analysis.

\section{Statistical analysis}

Procedures of SAS software (SAS ${ }^{\circledR}$ 9.4; SAS Institute Inc., Cary, NC, USA) were used to perform all statistical analyses and calculations. To test the differences between pretreatment and posttreatment scores, we performed the signed-rank test, a nonparametric test for dependent samples, utilizing 

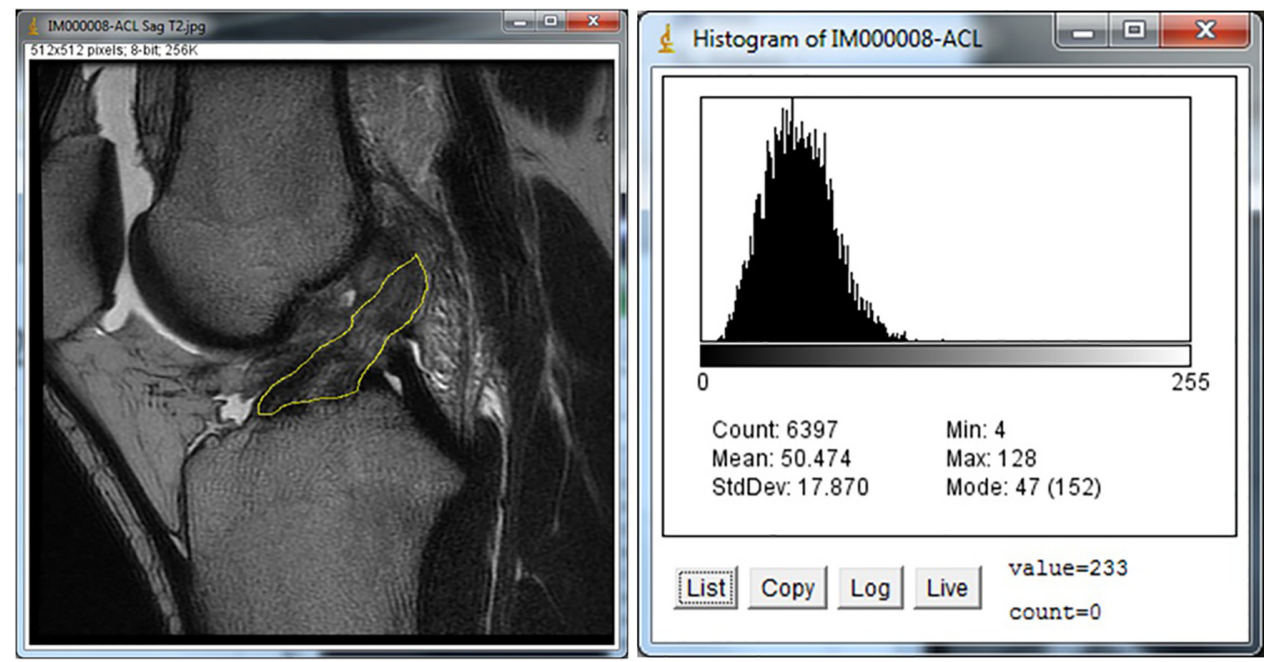

Figure $2 \mathrm{ACL}$ tracing and resulting histogram.

Abbreviations: $A C L$, anterior cruciate ligament; max, maximum; min, minimum; StdDev, standard deviation.

the UNIVARIATE procedure. Intraclass correlation was used to assess inter- and intrarater agreement of the ImageJ analyses. $P$-values of $\leq 0.05$ were considered as statistically significant.

\section{Results}

All of ten enrolled patients completed both pre- and posttreatment MRIs, six males and four females. The age range at the time of treatment was $16-52$ years. The mean time from injury to treatment was 3.45 months (range 1-11 months). Nine of ten patients received the injection during the subacute injury phase (range 1-4 months), and the tenth patient was treated 11 months after injury. There were five patients with grade 3 lesions, three with grade 2, and two with grade 1 ACL injuries, based on MRI assessment. The mean time to posttreatment MRI follow up was 3.7 months (range 2.5-7 months). The median time of the last observed

Table I NC count and volume injected for each patient

\begin{tabular}{lll}
\hline Patient & $\begin{array}{l}\text { NC count } \\
\text { (millions) }\end{array}$ & $\begin{array}{l}\text { Volume } \\
\text { injected }(\mathbf{m L})\end{array}$ \\
\hline A & 679 & 2.3 \\
B & 1123 & 4.8 \\
C & 375.6 & 2.6 \\
D & 567.8 & 2.2 \\
E & 716.3 & 2.4 \\
F & 841.5 & 3 \\
G & 1098 & 4 \\
H & 554.3 & 2 \\
I & 546.08 & 2.3 \\
J & 438.1 & 1.1 \\
All patients (mean $\pm S D)$ & $694 \pm 257$ & $2.7 \pm 1$ \\
\hline
\end{tabular}

Abbreviations: NC, nucleated cell; SD, standard deviation. outcome follow up was 6 months (range 3-24 months). The mean nucleated cell count and volume of the BMC was 694 million and $2.7 \mathrm{~mL}$, respectively (Table 1).

The intra- and interrater correlations for the selected metrics were excellent, ranging from 0.9 to 1 . Based on the histogram analysis of the pre- and posttreatment sagittal ACL MRIs, eight of ten patients demonstrated a decrease in the mean gray value, eight of ten had a decrease in the modal gray value, seven of ten had a decrease in the median, and ten of ten had a decrease in the raw integrated density. The decrease in the mean, median, and raw integrated density were all significant (Table 2). The decrease in the modal gray value was not significant $(P=0.08)$. Five of ten patients had a more positive skew (histograms leaned toward darker pixels), but this was not significant for the group $(P=0.6)$ (Figures 3-5).

Seven of ten patients completed all pre- and postprocedural outcome measures. Nine of ten completed the percentage change, seven of ten completed pain VAS, while seven of eight completed the LEFS, and one of two completed the FRI. The mean percentage change was $86.7 \%$ improved (range $40 \%-100 \%$ ). The mean pretreatment VAS score was 2.6 , and the mean VAS score posttreatment was 0.9 , with a mean pain change of -1.7 (range -6 to +2 ). The mean LEFS change was an improvement of 23.3 (range 5-38) points, and the single patient who completed the FRI reported an improvement of a decrease of 20 points (Table 3). LEFS score changes $(\mathrm{N}=6$, difference $=23.3)$ were statistically significant (signed-rank $P$-value $=0.0313$ ); however pain changes $(\mathrm{N}=7$, difference $=-1.7)$ did not show statistical significance (signed-rank $P$-value $=0.25$ ). The FRI is a scale 
Table 2 Pre- to posttreatment histogram measurement change statistical analysis

\begin{tabular}{lllllllll}
\hline Variable & Mean & SD & Median & Lower quartile & Upper quartile & Minimum & Maximum & $P$-value \\
\hline Image mean & -12.911 & 14.699 & -9.390 & -18.962 & -4.161 & -46.373 & 1.459 & 0.0098 \\
Image mode & -13.733 & 20.425 & -9.167 & -24.667 & -4.333 & -45.333 & 17.000 & 0.084 \\
Image median & -12.367 & 14.358 & -9.833 & -18.333 & 0 & -42.333 & 3.000 & 0.0195 \\
Skewness & 0.243 & 0.529 & -0.024 & -0.093 & 0.422 & -0.292 & 1.204 & 0.625 \\
Raw Integrated & -100783 & 77363 & -71590 & -163967 & -48921 & -244398 & -3112 & 0.002 \\
Density & & & & & & & & \\
\hline
\end{tabular}

Note: $P$-values were calculated using the signed-rank test.

of $0-100$, with lower score indicating better function, ${ }^{26}$ and the LEFS is a scale of $0-80$, with a higher score indicating better function. ${ }^{27}$ The meaningful detectable change in the FRI is $12.3,{ }^{28}$ and the minimal detectable change and the minimally important change for the LEFS is $9,{ }^{27}$ thus based on those criteria, our reported changes in function are meaningful.

\section{Discussion}

The normal ACL ligament appears with a low signal (dark), and an injured or disrupted ligament shows a high signal (bright) on most MRI sequences. ${ }^{25,29}$ However, interpreting some findings on an MRI can be a subjective process, with substantial variability between readers. ${ }^{30}$ The sagittal image was selected for the ROI evaluation because it is most helpful

A Patient H Pre MRI and Histogram
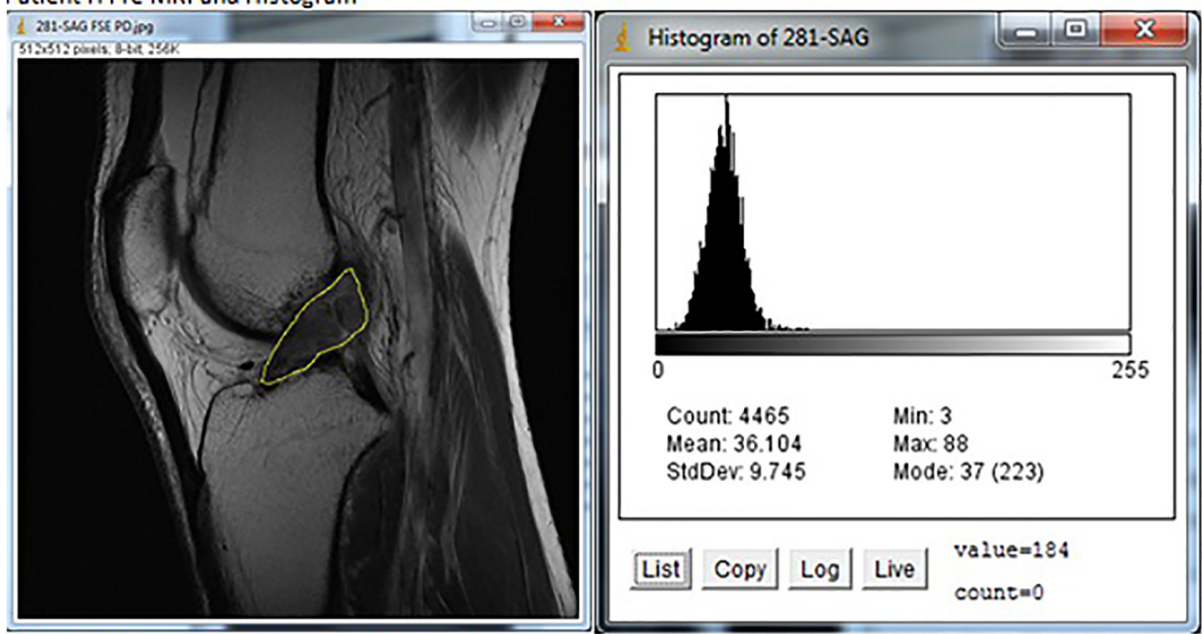

B
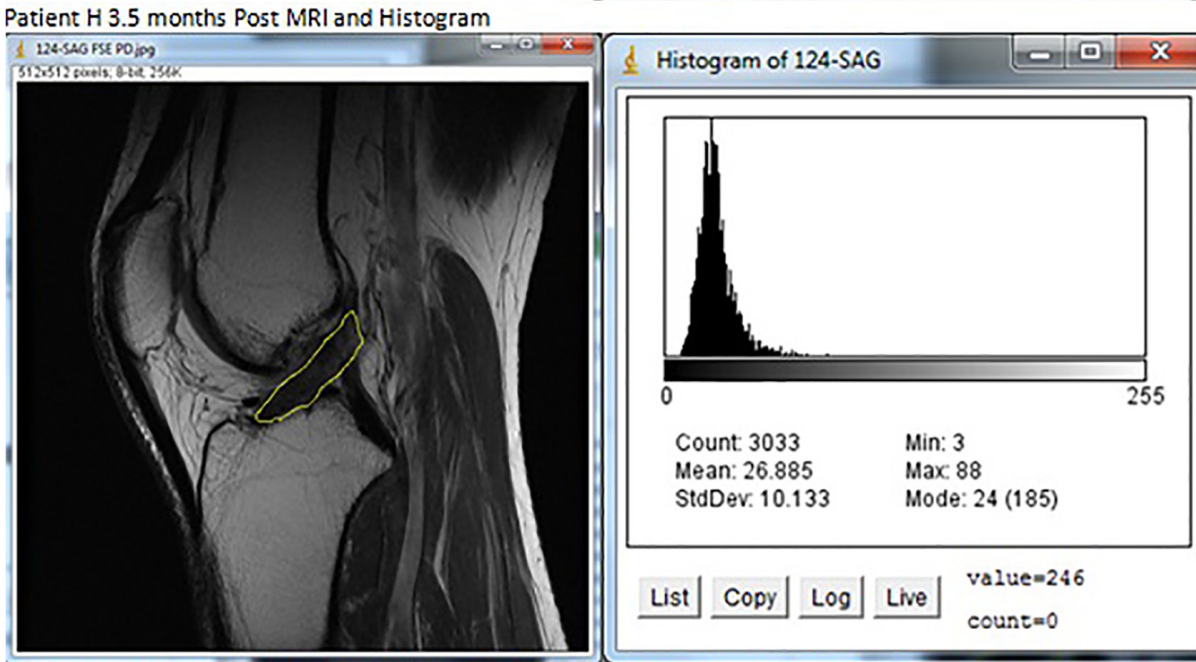

Count: 3033

Mean: 26.885

StdDev. 10.133

Max 88

Mode: 24 (185)

List Copy Log Live

value $=246$

count $=0$

Figure 3 MRIs and histograms for Patient $\mathrm{H}$, (A) pre- and (B) at 3.5 months posttreatment.

Note: A darker improved ACL image was seen on the posttreatment MRI, as well as the leftward shift of pixels toward darker values in the graph.

Abbreviations: $A C L$, anterior cruciate ligament; max, maximum; min, minimum; MRI, magnetic resonance imaging; StdDev, standard deviation. 


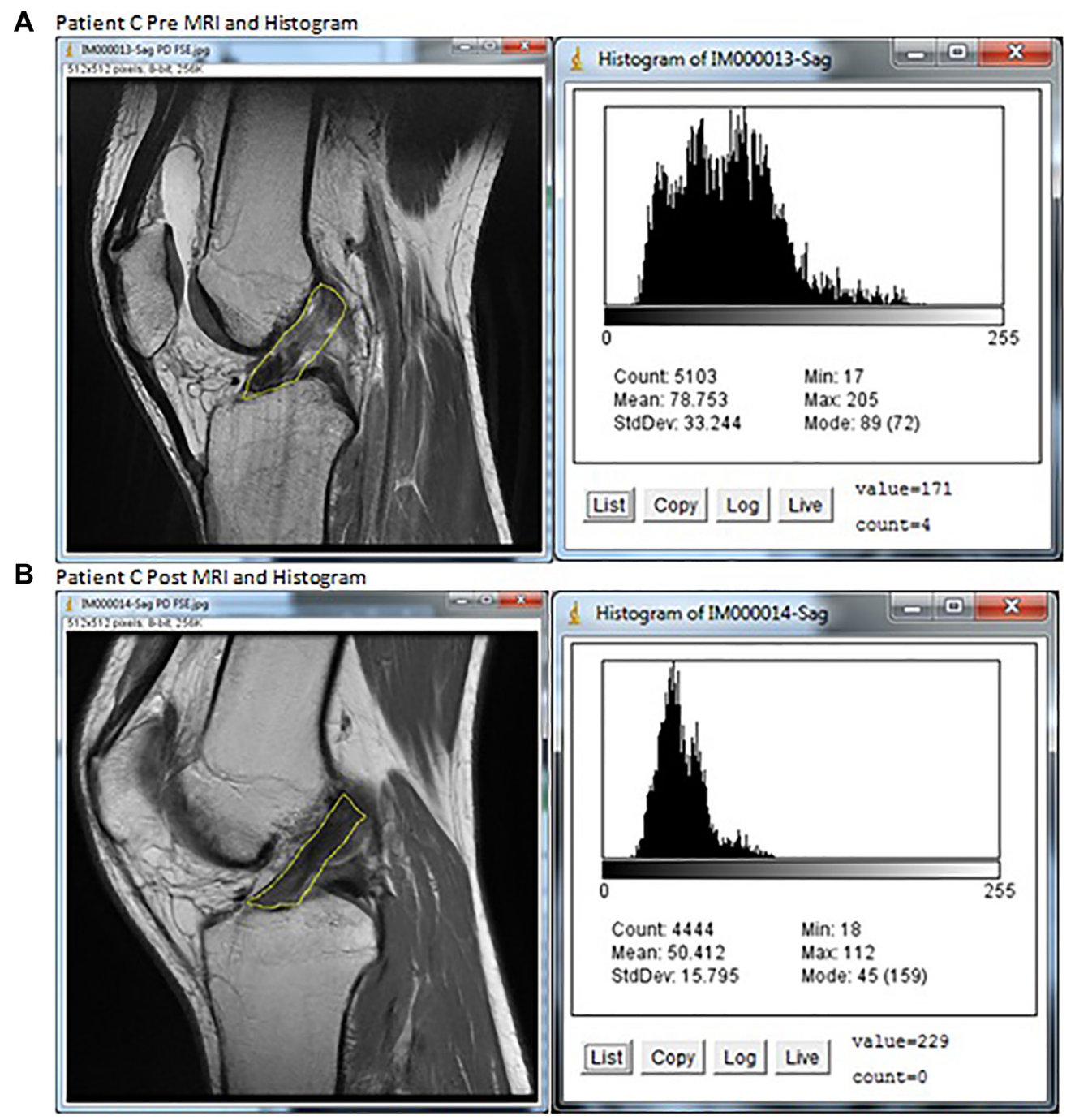

Figure 4 MRls and histograms for Patient C, (A) pre- and (B) posttreatment.

Note: A darker improved ACL image was seen on the posttreatment MRI, as well as the leftward shift of pixels toward darker values in the graph.

Abbreviations: $A C L$, anterior cruciate ligament; max, maximum; min, minimum; MRI, magnetic resonance imaging; StdDev, standard deviation.

in the evaluation of the linear integrity of ACL fibers..$^{25}$ The ROI protocol and ImageJ software analysis demonstrated excellent inter- and intrarater reliability.

The five metrics used to quantify the gray scale of the ROI are explained as follows: 1) the mean gray value is the sum of the gray values of all the pixels in the selection divided by the number of pixels; 2) the modal gray value is the most frequently occurring gray value within the selection and corresponds to the highest peak in the histogram; 3 ) the median is the middle value of all of the pixels in the selection, with lower value correlating with a darker image; 4) skewness describes how the histogram is distributed to one or the other side of the mean, with a positive skewness indicating the right tail is longer than the left, and the mass of the distribution concentrated on the left indicating a darker image; and 5) the raw integrated density is the sum of the values of the pixels in the selection, with a lower value an indication of a darker image.

In this case study, seven of ten patients demonstrated improvement in at least four of five measures of pixel intensity in their ACLs from pre- to posttreatment, in the expected direction of a darker and more normal appearing ACL. All five of the objective measures showed a trend toward darker ACLs, from pre- to posttreatment MRIs (more normal appearing), and three of five reached $95 \%$ statistical significance.

Subjectively, all of the nine patients that completed Likert percentage changes reported improvement. Eight of those patients reported $70 \%$ or greater improvement. All seven patients that completed both pre- and posttreatment functional scales (LEFS or FRI) reported improvement that was clinically meaningful per the metrics of those individual 
A Patient B pre MRI and histogram
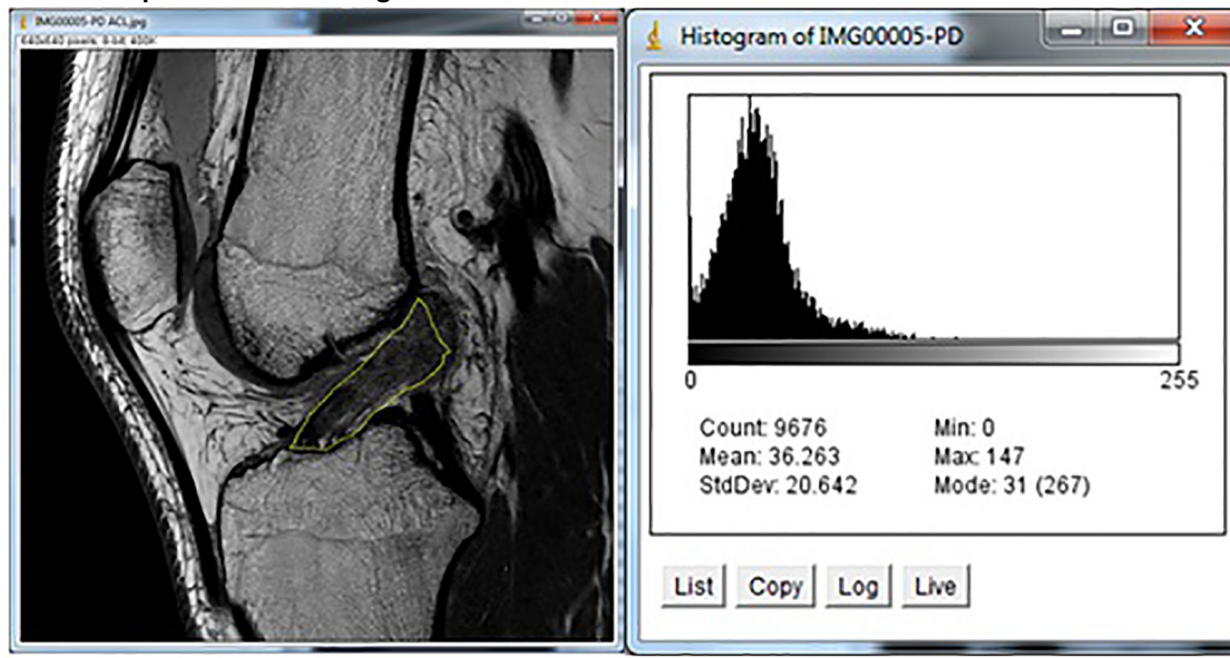

List Copy Log Live

B Patient B post MRI and histogram
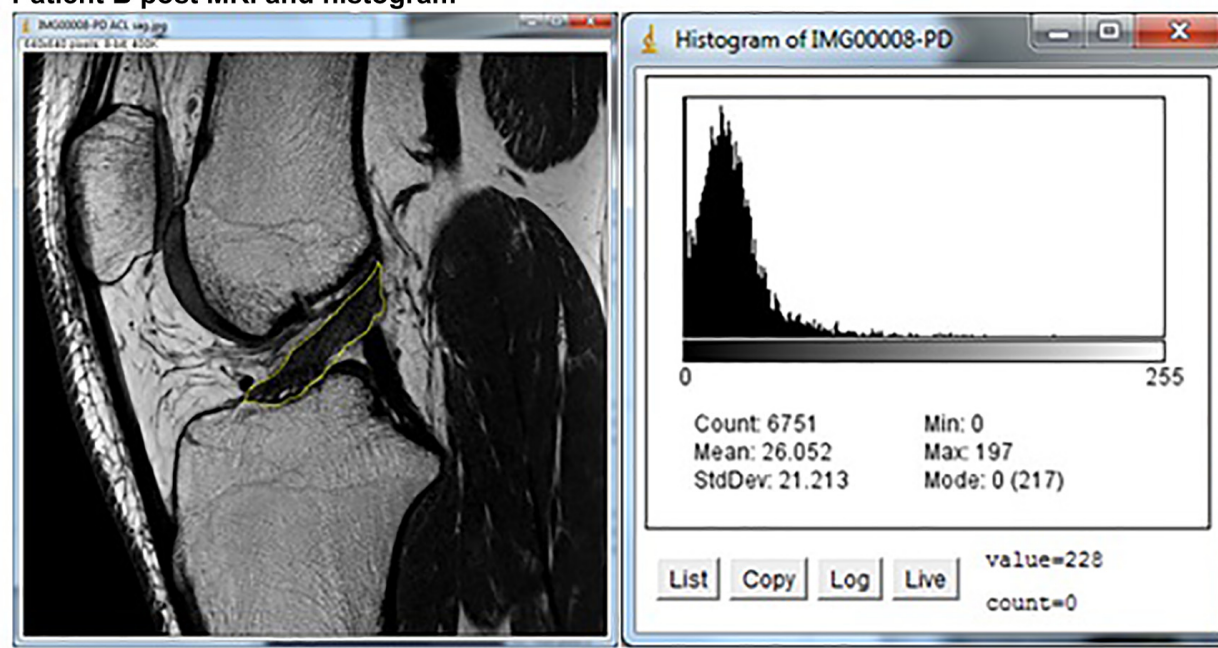

List Copy Log Live $\begin{aligned} & \text { value }=228 \\ & \text { count }=0\end{aligned}$

Figure 5 MRIs and histograms for Patient B, (A) pre- and (B) posttreatment.

Note: A darker improved ACL image was seen on the posttreatment MRI, as well as the leftward shift of pixels toward darker values in the graph.

Abbreviations: ACL, anterior cruciate ligament; max, maximum; min, minimum; MRI, magnetic resonance imaging; StdDev, standard deviation.

Table 3 Patient clinical outcomes

\begin{tabular}{|c|c|c|c|c|c|c|c|}
\hline Patient & $\begin{array}{l}\text { ACL tear } \\
\text { grade }\end{array}$ & $\begin{array}{l}\text { Likert \% } \\
\text { change }\end{array}$ & $\begin{array}{l}\text { LEFS* } \\
\text { pretreatment }\end{array}$ & $\begin{array}{l}\text { LEFS } \\
\text { posttreatment }\end{array}$ & $\begin{array}{l}\text { Pain } \\
\text { pretreatment }\end{array}$ & $\begin{array}{l}\text { Pain } \\
\text { posttreatment }\end{array}$ & $\begin{array}{l}\text { Months } \\
\text { postprocedure }\end{array}$ \\
\hline A & 3 & 70 & 40 & 64 & 5 & 3 & 6 \\
\hline B & 2 & 100 & 41 & 79 & 4 & 0 & 6 \\
\hline C & 2 & $100^{\#}$ & 30 & 38 & I & 3 & 3 \\
\hline D & 3 & NA & 53 & NA & 2 & NA & NA \\
\hline$E$ & I & 100 & 75 & 80 & 0 & 0 & 6 \\
\hline $\mathrm{F}$ & 2 & 80 & 48 & 75 & 6 & 0 & 12 \\
\hline G & 3 & 90 & 41 & 79 & 2 & 0 & 6 \\
\hline Patient & $\begin{array}{l}\text { ACL tear } \\
\text { grade }\end{array}$ & $\begin{array}{l}\text { Likert \% } \\
\text { change }\end{array}$ & $\begin{array}{l}\text { FRI* } \\
\text { pretreatment }\end{array}$ & $\begin{array}{l}\text { FRI posttreatment } \\
\text { (1 } 2 \text { mon) }\end{array}$ & $\begin{array}{l}\text { Pain } \\
\text { pretreatment }\end{array}$ & $\begin{array}{l}\text { Pain } \\
\text { posttreatment }\end{array}$ & $\begin{array}{l}\text { Months } \\
\text { postprocedure }\end{array}$ \\
\hline $\mathrm{H}$ & 3 & 100 & 22.5 & 2.5 & 0 & 0 & 24 \\
\hline I & I & 40 & NA & 62 & NA & 3 & 3 \\
\hline J & 3 & 100 & NA & NA & NA & NA & 18 \\
\hline
\end{tabular}

Notes: *FRI was scored on a scale of 0-100 (lowest is best function); LEFS was scored on a scale of 0-80 (highest is the best function); " Likert reporting was missing at 3 months, but the patient reported 100\% improvement at 12 months.

Abbreviations: ACL, anterior cruciate ligament; FRI, Functional Rating Index; LEFS, Lower Extremity Functional Scale; NA, not available. 
functional questionnaires. The functional scales changed through the course of the study because these patients were tracked as part of an ongoing registry that tracks many patients with various orthopedic injuries. The decision to change the questionnaire was based on the evolution of the needs of that larger data collection project, and the LEFS is one of many validated lower-extremity functional questionnaires. This only affected one patient, so it is unlikely to have affected the interpretation of the results. However the use of two different functional questionnaires may skew interpretation of the functional data due to lack of a consistent method of functional data reporting.

In this study, the majority of patients (five of ten) had grade 3 tears. We believe the significance of this finding is that grade 3 tears are more likely to be disabling due to the complete rupture, and thus, these patients are more likely to seek help. We did treat two injuries that were classified as nonhealing grade 1 tears, which theoretically could have spontaneously healed without treatment. We excluded patients with ligament retraction greater than $1 \mathrm{~cm}$ because of concern that the MSCs might not be able to bridge a gap between a tear any larger, which was inferred from clinical experience gained in treating these cases. We believe that a $1 \mathrm{~cm}$ or greater gap would not respond to biological treatment and would be a better candidate for surgical treatment.

It should be noted that the specific treatment protocol used in this study is not standard or widely accepted and is unique to this specific center and affiliated clinics. It was uniquely designed based on our expert opinion. The rationale for the treatment protocol chosen was to provide a proinflammatory and proliferative stimulus with a preinjection and then several days later, once the cellular phase of healing had been initiated, to augment the area with BMC rich in MSCs and $\mathrm{PRP} /$ platelet growth factors, and then finally, to provide $\mathrm{PRP} /$ platelet growth factors to promote MSC survival. Research has suggested that hypertonic dextrose can provide a proliferative stimulus to ligaments and tendons. ${ }^{31,32}$ It has also been shown through multiple in vitro studies that PRP can stimulate and provide supportive nutrients to MSCs. ${ }^{33-35}$ Similarly, PL, the product of stripping viable growth factors from whole platelets, can also promote MSC proliferation and support these cells in culture. ${ }^{35-37}$ We used both intraligamentous and intraarticular sites due to the common finding of osteoarthritis after ACL injury, ${ }^{38}$ theorizing that the IA injection may help undetected chondral injury. Given that there are many variables that remained uncontrolled, this limits the validity and generalizability of these results outside of the use of this specific protocol.

Postprocedural rehabilitation instructions included activity as tolerated. Our goal was to allow the patient to load the ligament, depending on pain. Past study has shown that loading is essential for ligament healing. ${ }^{39}$ Physical activity and physical therapy programs were not controlled. We are unaware of any specific physical therapy or physical activity research demonstrating a benefit for healing ACL tears. Some physicians may have recommended bracing postprocedure as well. Given that bracing rarely allows full healing of an ACL on MRI, based on our clinical experience, we did not feel that bracing (or not bracing) would make a difference. However, being the traditional approach for conservative care in ACL injuries, some would argue that we might have seen better results had we required bracing, and the lack of standardized rehabilitation is a limitation of the study.

For imaging analysis, we searched for an open-source imaging analysis program that had histogram capabilities. It should also be noted that despite excellent inter- and intrarater reliability, the NIH ImageJ software has not been used in a similar study involving ligament changes on MRI. However, it has been used in other areas of medicine requiring histogrambased analysis of medical imaging. ${ }^{40-44}$ At the time of this manuscript was being prepared, there were 789 studies that came up under the search term "ImageJ" on PubMed. Finally, the use of two different functional questionnaires may have skewed interpretation of the functional data, due to lack of a consistent method of functional data reporting.

Major limitations of the study include the lack of a control group and the lack of randomization. As a result, we cannot conclude that the observed effects were due to the MSC treatment versus natural healing or the pretreatment protocol. There is good reason to believe that the results were probably due to the MSC therapy, however. Grade 2-3 ACL injuries rarely heal spontaneously. ${ }^{45-47}$ It is possible that patients could have reported improved symptoms because they adapted to the instability, because of placebo effect, or because of some natural healing that may have reduced symptoms.

Given the specific treatment protocol with multiple variables, any component of the protocol could have accounted for the observed effects. We do not believe that the pretherapy injection routine, which was meant to cause a proinflammatory healing environment, was the cause of the observed changes. There is no evidence that we are aware of that the injection of a $12.5 \%$ hypertonic dextrose solution can result in objective improvements in the MRI appearance of ligaments. 
While it could be argued that it was the PRP component of the MSC injectate that was the cause of the observed effects, the results of prior studies do not support such a conclusion. PRP use has been previously studied in patients undergoing ACL reconstruction, with one study showing increased cortical bone formation on $\mathrm{MRI}^{48}$ and another describing faster remodeling of the grafts. ${ }^{49} \mathrm{~A}$ third study did not report any differences on posttreatment MRI.$^{50}$ We were unable to find any studies demonstrating a benefit of PRP for ACL injuries, however. Lastly, the lack of pre- and posttreatment objective measurements of ACL laxity, such as KT1000 testing, ${ }^{51}$ is a major limitation in accessing treatment outcomes.

For future study, the use of a larger randomized, controlled trial would be warranted. We suggest recorded clinical findings before and after treatment, such as the Lachman, pivot shift test, an objective ligament integrity test (eg, Rolimeter or KT1000 test), and a validated functional questionnaire. Postprocedural rehabilitation should be standardized as well. In addition to the objective review of ACL integrity on MRI with software, review of pre- and posttreatment MRIs should ideally be performed at one center, with a blinded musculoskeletal radiologist reviewing the images and reporting findings. With a larger number, perhaps statistical analysis of the difference in outcome between patients with varying grades of ACL tear would shed light on which patients may be more or less likely to benefit from this type of treatment.

\section{Conclusion}

Based on this small case series, the precise injection of autologous bone marrow-derived nucleated cells into the ACL ligament under fluoroscopic guidance, utilizing this specific protocol shows promise as a treatment for symptomatic grade 1, 2, and, possibly, nonretracted grade 3 ACL tears. Further investigation is warranted by these initial encouraging results. A well-populated randomized, controlled trial is needed to determine whether our results are repeatable and due to the intervention used in this study.

\section{Acknowledgments}

John Schultz, MD, Ron Hanson, MD, and Benjamin Newton, MD all participated in treating patients. Dr Newton also read images for interrater reliability testing.

\section{Disclosure}

John Pitts and Christopher J Centeno are affiliated with the Centeno-Schultz Clinic. Dr Centeno is also a shareholder and director of Regenerative Sciences, LLC. Michael D Freeman is affiliated with Oregon Health and Science University. Hasan Al-Sayegh is employed by Regenerative Sciences, LLC. The authors report no other conflicts of interest.

\section{References}

1. Whitehead TS. Failure of anterior cruciate ligament reconstruction. Clin Sports Med. 2013;32(1):177-204.

2. Seijas R, Ares O, Cuscó X, Alvarez P, Steinbacher G, Cugat R. Partial anterior cruciate ligament tears treated with intraligamentary plasma rich in growth factors. World J Orthop. 2014;5(3):373-378.

3. Pujol N, Colombet P, Cucurulo T, et al; French Arthroscopy Society (SFA). Natural history of partial anterior cruciate ligament tears: a systematic literature review. Orthop Traumatol Surg Res. 2012; 98(8 Suppl):S160-S164.

4. Georgoulis AD, Ristanis S, Moraiti CO, et al. ACL injury and reconstruction: Clinical related in vivo biomechanics. Orthop Traumatol Surg Res. 2010;96(8 Suppl):S119-S128.

5. Vavken P, Murray MM. Translational studies in anterior cruciate ligament repair. Tissue Eng Part B Rev. 2010;16(1):5-11.

6. Fisher MB, Liang R, Jung HJ, et al. Potential of healing a transected anterior cruciate ligament with genetically modified extracellular matrix bioscaffolds in a goat model. Knee Surg Sports Traumatol Arthrosc. 2012;20(7):1357-1365.

7. Vavken P, Murray MM. The potential for primary repair of the ACL. Sports Med Arthrosc. 2011;19(1):44-49.

8. Kanaya A, Deie M, Adachi N, Nishimori M, Yanada S, Ochi M. Intraarticular injection of mesenchymal stromal cells in partially torn anterior cruciate ligaments in a rat model. Arthroscopy. 2007;23(6):610-617.

9. Wang H, Fleischli JE, Hutchinson ID, Zheng NN. Knee moment and shear force are correlated with femoral tunnel orientation after singlebundle anterior cruciate ligament reconstruction. Am J Sports Med. 2014;42(10):2377-2385.

10. Li RT, Lorenz S, Xu Y, Harner CD, Fu FH, Irrgang JJ. Predictors of radiographic knee osteoarthritis after anterior cruciate ligament reconstruction. Am J Sports Med. 2011;39(12):2595-2603.

11. Sampson S, Botto-van Bemden A, Aufiero D. Autologous bone marrow concentrate: review and application of a novel intra-articular orthobiologic for cartilage disease. Phys Sportsmed. 2013;41(3):7-18.

12. Paterno MV, Schmitt LC, Ford KR, et al. Biomechanical measures during landing and postural stability predict second anterior cruciate ligament injury after anterior cruciate ligament reconstruction and return to sport. Am J Sports Med. 2010;38(10):1968-1978.

13. Centeno CJ, Schultz JR, Cheever M, et al. Safety and complications reporting update on the re-implantation of culture-expanded mesenchymal stem cells using autologous platelet lysate technique. Curr Stem Cell Res Ther. 2011;6(4):368-378.

14. Centeno CJ, Busse D, Kisiday J, Keohan C, Freeman M, Karli D. Increased knee cartilage volume in degenerative joint disease using percutaneously implanted, autologous mesenchymal stem cells. Pain Physician. 2008;11(3):343-353.

15. Centeno CJ, Busse D, Kisiday J, Keohan C, Freeman M, Karli D. Regeneration of meniscus cartilage in a knee treated with percutaneously implanted autologous mesenchymal stem cells. Med Hypotheses. 2008;71(6):900-908.

16. Rudolph KS, Eastlack ME, Axe MJ, Snyder-Mackler L. 1998 Basmajian Student Award Paper: Movement patterns after anterior cruciate ligament injury: a comparison of patients who compensate well for the injury and those who require operative stabilization. $J$ Electromyogr Kinesiol. 1998;8(6):349-362.

17. Lerat JL, Moyen BL, Cladiere F, Besse JL, Abidi H. Knee instability after injury to the anterior cruciate ligament. Quantification of the Lachman test. J Bone Joint Surg Br. Jan 2000;82(1):42-47.

18. Wasilewski SA, Covall DJ, Cohen S. Effect of surgical timing on recovery and associated injuries after anterior cruciate ligament reconstruction. Am J Sports Med. 1993;21(3):338-342. 
19. Hong SH, Choi JY, Lee GK, Choi JA, Chung HW, Kang HS. Grading of anterior cruciate ligament injury. Diagnostic efficacy of oblique coronal magnetic resonance imaging of the knee. J Comput Assist Tomogr. 2003;27(5):814-819.

20. Chen MR, Dragoo JL. The effect of nonsteroidal anti-inflammatory drugs on tissue healing. Knee Surg Sports Traumatol Arthrosc. 2013; 21(3):540-549.

21. Wiggins ME, Fadale PD, Barrach H, Ehrlich MG, Walsh WR. Healing characteristics of a type I collagenous structure treated with corticosteroids. Am J Sports Med. 1994;22(2):279-288.

22. Tsai WC, Hsu CC, Chen CP, Chen MJ, Lin MS, Pang JH. Ibuprofen inhibition of tendon cell migration and down-regulation of paxillin expression. J Orthop Res. 2006;24(3):551-558.

23. Tsai WC, Tang FT, Wong MK, Pang JH. Inhibition of tendon cell migration by dexamethasone is correlated with reduced alpha-smooth muscle actin gene expression: a potential mechanism of delayed tendon healing. J Orthop Res. 2003;21(2):265-271.

24. Randelli P, Randelli F, Cabitza P, Vaienti L. The effects of COX-2 antiinflammatory drugs on soft tissue healing: a review of the literature. $J$ Biol Regul Homeost Agents. 2010;24(2):107-114.

25. Roberts CC, Towers JD, Spangehl MJ, Carrino JA, Morrison WB. Advanced MR imaging of the cruciate ligaments. Radiol Clin North Am. 2007;45(6):1003-1016.

26. Feise RJ, Michael Menke J. Functional rating index: a new valid and reliable instrument to measure the magnitude of clinical change in spinal conditions. Spine (Phila Pa 1976). 2001;26(1):78-86; discussion 87.

27. Binkley JM, Stratford PW, Lott SA, Riddle DL. The Lower Extremity Functional Scale (LEFS): scale development, measurement properties, and clinical application. North American Orthopaedic Rehabilitation Research Network. Phys Ther. 1999;79(4):371-383.

28. Childs JD, Piva SR. Psychometric properties of the functional rating index in patients with low back pain. Eur Spine J. 2005;14(10):1008-1012.

29. Pope TL Jr. MRI of knee ligaments. Semin Ultrasound CT MR. 1994;15(5):366-382.

30. Krampla W, Roesel M, Svoboda K, Nachbagauer A, Gschwantler M, Hruby W. MRI of the knee: how do field strength and radiologist's experience influence diagnostic accuracy and interobserver correlation in assessing chondral and meniscal lesions and the integrity of the anterior cruciate ligament? Eur Radiol. 2009;19(6):1519-1528.

31. Yoshii Y, Zhao C, Schmelzer JD, Low PA, An KN, Amadio PC. The effects of hypertonic dextrose injection on connective tissue and nerve conduction through the rabbit carpal tunnel. Arch Phys Med Rehabil. 2009;90(2):333-339.

32. Freeman JW, Empson YM, Ekwueme EC, Paynter DM, Brolinson PG. Effect of prolotherapy on cellular proliferation and collagen deposition in MC3T3-E1 and patellar tendon fibroblast populations. Transl Res. 2011;158(3):132-139.

33. Amable PR, Teixeira MV, Carias RB, Granjeiro JM, Borojevic R. Mesenchymal stromal cell proliferation, gene expression and protein production in human platelet-rich plasma-supplemented media. PLoS One. 2014;9(8):e104662

34. Lee JK, Lee S, Han SA, Seong SC, Lee MC. The effect of platelet-rich plasma on the differentiation of synovium-derived mesenchymal stem cells. J Orthop Res. 2014;32(10):1317-1325.
35. Mojica-Henshaw MP, Jacobson P, Morris J, et al. Serum-converted platelet lysate can substitute for fetal bovine serum in human mesenchymal stromal cell cultures. Cytotherapy. 2013;15(12):1458-1468.

36. Iudicone P, Fioravanti D, Bonanno G, et al. Pathogen-free, plasma-poor platelet lysate and expansion of human mesenchymal stem cells. JTransl Med. 2014;12:28.

37. Shanskii YD, Sergeeva NS, Sviridova IK, et al. Human platelet lysate as a promising growth-stimulating additive for culturing of stem cells and other cell types. Bull Exp Biol Med. 2013;156(1):146-151.

38. Louboutin H, Debarge R, Richou J, et al. Osteoarthritis in patients with anterior cruciate ligament rupture: a review of risk factors. Knee. 2009;16(4):239-244.

39. Hsu WH, Peng KT, Lai LJ, Hung CH, Chang PJ. Cellular senescence occurring in the rabbit medial collateral ligament during healing. J Orthop Res. 2013;31(1):81-90.

40. Natarajan S, Juneja M, Pallam NK, Boaz K, Mohindra A, Lewis A. A novel technique to assess chromatin texture using pixel optical densitometry in oral squamous cell carcinoma. Microsc Res Tech. 2012;75(8):1119-1123.

41. Chou SY, Chen CY, Su HW, Hsu MI, Liang SR, Hsu CS. Ultrasonographic quantification of the endometrium during the menstrual cycle using computer-assisted analysis. Taiwan J Obstet Gynecol. 2011;50(3):297-300.

42. Kim US, Kim SJ, Baek SH, Kim HK, Sohn YH. Quantitative analysis of optic disc color. Korean J Ophthalmol. 2011;25(3):174-177.

43. Meier A, Farrow C, Harris BE, King GG, Jones A. Application of texture analysis to ventilation SPECT/CT data. Comput Med Imaging Graph. 2011;35(6):438-450.

44. Chou SY, Chen CY, Chow PK, Hsu CS, Hsu MI, Chiang HK. Ultrasonographic evaluation of endometrial changes using computer assisted image analysis. J Obstet Gynaecol Res. 2010;36(3):634-638.

45. Rizzello G, Longo UG, Petrillo S, et al. Growth factors and stem cells for the management of anterior cruciate ligament tears. Open Orthop J. 2012;6:525-530.

46. Woo SL, Niyibizi C, Matyas J, Kavalkovich K, Weaver-Green C, Fox RJ. Medial collateral knee ligament healing. Combined medial collateral and anterior cruciate ligament injuries studied in rabbits. Acta Orthop Scand. 1997;68(2):142-148.

47. Bray RC, Leonard CA, Salo PT. Correlation of healing capacity with vascular response in the anterior cruciate and medial collateral ligaments of the rabbit. J Orthop Res. 2003;21(6):1118-1123.

48. Rupreht M, Vogrin M, Hussein M. MRI evaluation of tibial tunnel wall cortical bone formation after platelet-rich plasma applied during anterior cruciate ligament reconstruction. Radiol Oncol. 2013;47(2):119-124.

49. Seijas R, Ares O, Catala J, Alvarez-Diaz P, Cusco X, Cugat R. Magnetic resonance imaging evaluation of patellar tendon graft remodelling after anterior cruciate ligament reconstruction with or without platelet-rich plasma. J Orthop Surg (Hong Kong). 2013;21(1):10-14.

50. Silva A, Sampaio R. Anatomic ACL reconstruction: does the plateletrich plasma accelerate tendon healing? Knee Surg Sports Traumatol Arthrosc. 2009;17(6):676-682.

51. Rangger C, Daniel DM, Stone ML, Kaufman K. Diagnosis of an ACL disruption with KT-1000 arthrometer measurements. Knee Surg Sports Traumatol Arthrosc. 1993;1(1):60-66
Journal of Pain Research

\section{Publish your work in this journal}

The Journal of Pain Research is an international, peer-reviewed, open access, online journal that welcomes laboratory and clinical findings in the fields of pain research and the prevention and management of pain. Original research, reviews, symposium reports, hypothesis formation and commentaries are all considered for publication.

\section{Dovepress}

The manuscript management system is completely online and includes a very quick and fair peer-review system, which is all easy to use. Visit http://www.dovepress.com/testimonials.php to read real quotes from published authors. 